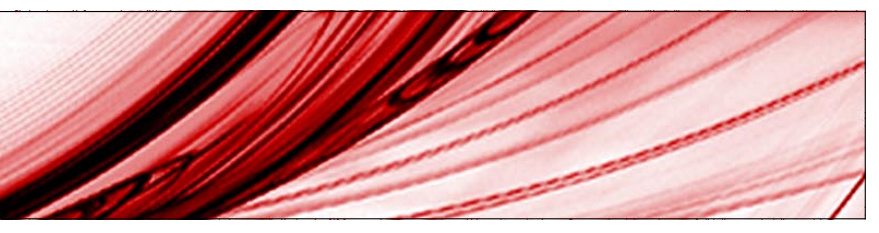

\title{
Adaptive node-to-node pinning synchronization control of complex networks
}

Luiz Felipe R. Turci and Elbert. E. N. Macau

Citation: Chaos 22, 033151 (2012); doi: 10.1063/1.4754436

View online: http://dx.doi.org/10.1063/1.4754436

View Table of Contents: http://chaos.aip.org/resource/1/CHAOEH/v22/i3

Published by the American Institute of Physics.

\section{Related Articles}

A novel synchronization scheme with a simple linear control and guaranteed convergence time for generalized Lorenz chaotic systems

Chaos 22, 043108 (2012)

Transmission projective synchronization of multi-systems with non-delayed and delayed coupling via impulsive control

Chaos 22, 043107 (2012)

Robustness to noise in synchronization of network motifs: Experimental results

Chaos 22, 043106 (2012)

On the formulation and solution of the isochronal synchronization stability problem in delay-coupled complex networks

Chaos 22, 033152 (2012)

An analytic criterion for generalized synchronization in unidirectionally coupled systems based on the auxiliary system approach

Chaos 22, $033146(2012)$

\section{Additional information on Chaos}

\section{Journal Homepage: http://chaos.aip.org/}

Journal Information: http://chaos.aip.org/about/about_the_journal

Top downloads: http://chaos.aip.org/features/most_downloaded

Information for Authors: http://chaos.aip.org/authors

\section{ADVERTISEMENT}

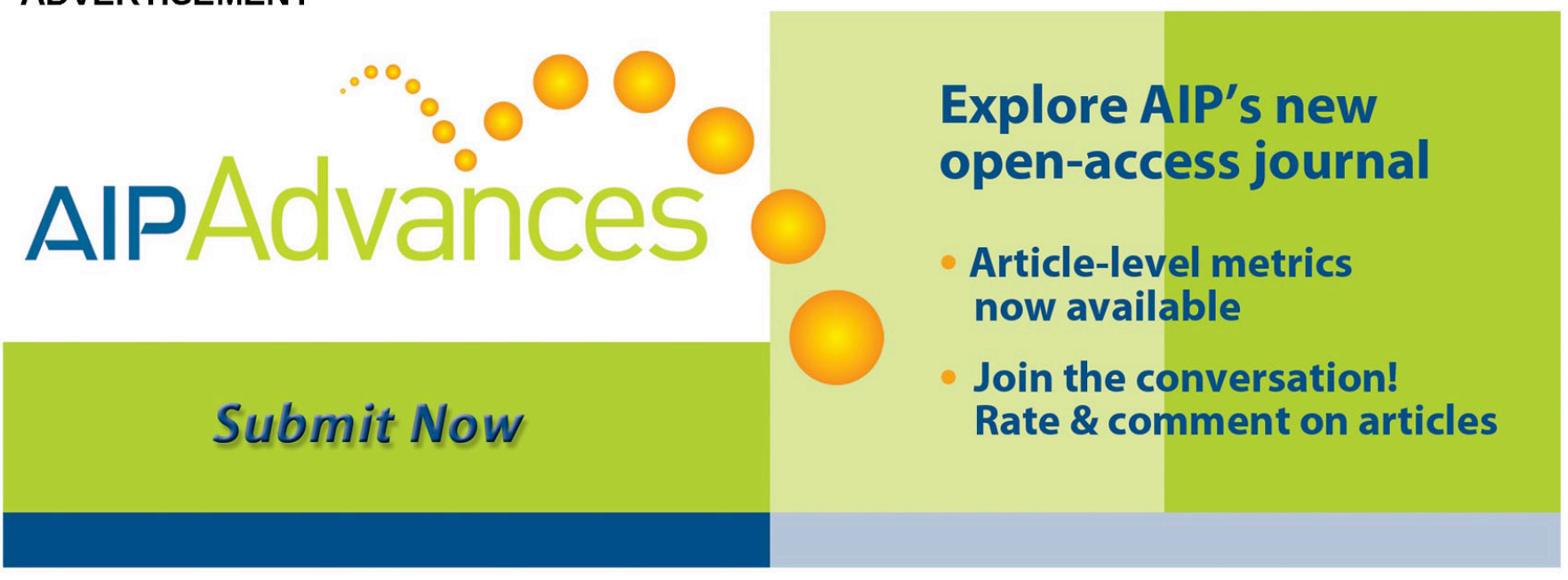




\title{
Adaptive node-to-node pinning synchronization control of complex networks
}

Luiz Felipe R. Turci ${ }^{1, \text { a) }}$ and Elbert. E. N. Macau ${ }^{2, b)}$

${ }^{1}$ Federal University of Alfenas-UNIFAL-MG, Poços de Caldas, Brazil

${ }^{2}$ National Institute for Space Research-INPE, São José dos Campos, Brazil

(Received 3 May 2012; accepted 5 September 2012; published online 27 September 2012)

\begin{abstract}
In this work, we propose an adaptive node-to-node pinning control strategy. In this approach, both the coupling strength among nodes and the pinning control gains are adaptively changed according to well chosen adaptation laws that take into account the specificities of the oscillators and the network topology. Proof of stability and performance comparison is also shown in this paper. (c) 2012 American Institute of Physics. [http://dx.doi.org/10.1063/1.4754436]
\end{abstract}

\begin{abstract}
A great variety of nowadays systems can be regarded as networks of interconnected chaotic dynamical agents, i.e., oscillators which interact with each other. Using this network description, the oscillators are located in nodes, while the edges of the network capture the interaction links among the oscillators. Also, researchers in different areas of applied science and engineering have addressed the problem of how to apply control strategies that exploit the network topology and the information exchange among the nodes to obtain desired ordered collective behaviors in the interconnected system. Examples of these control actions necessarily include rendezvous and flocking problems in robotics, ${ }^{1}$ synchronization of sensor networks, ${ }^{2-5}$ consensus and multi-agent coordination problems in control theory, ${ }^{6,7}$ the emergence of coordinated motion in animal behavior, and other biological systems (see, for instance, Refs. 8 and 9). In this work, we propose an adaptive node-tonode pinning control strategy to achieve control goals in distributed scenarios.
\end{abstract}

\section{INTRODUCTION}

Coordinated motion, ${ }^{3,9,10}$ consensus, ${ }^{7,11}$ and synchronization $^{12-15}$ are the key ingredients that account for the ordered behavior that appears in those previously cited situations. Consequently, the main point here is to find strategies to allow one to regulate oscillators' behavior and interactions among them so that all the oscillators evolve toward a same asymptotic behavior. When this takes place, the system is considered synchronized.

Consider a complex network in which the oscillators are identical and interconnected in a linear diffusive coupling configuration, i.e.,

$$
\dot{\mathbf{x}}_{i}(t)=f\left(\mathbf{x}_{i}(t), t\right)-\sigma \sum_{j \in \mathcal{N}_{i}} \Gamma\left\{\mathbf{H}\left(\mathbf{x}_{i}(t)\right)-\mathbf{H}\left(\mathbf{x}_{j}(t)\right)\right\},
$$

with $\mathbf{x}_{i}(t)=\left[x_{i 1}(t), \ldots, x_{i n}(t)\right]^{T}$ being the n-dimensional state vector of node $i, f\left(\mathbf{x}_{i}(t), t\right)$ the vector field describing node

\footnotetext{
a)felipeturci@yahoo.com.br.

b) elbert@lac.inpe.br.
}

dynamics, $\sigma$ a unique global coupling strength among nodes assumed to be constant and time-invariant, $\Gamma$ is the inner coupling matrix, and $\mathbf{H}$ is the coupling function that for linearly diffusive coupling is defined as $\mathbf{H}(\mathbf{x})=\mathbf{x} . \mathcal{N}_{i}$ is the set of neighbors of node $i$, that is, the set of nodes connected to node $i$. Given the node dynamics, the synchronization problem consists of finding on what range values of $\sigma$ the network synchronizes. Such problem, termed as the synchronizability problem, has been solved mainly by the use of the so-called master stability function (MSF) approach (first introduced in Ref. 16).

For situations in which the whole network is not synchronizable, according to MSF approach, controllers may be designed and applied to force the network to synchronize. However, considering efficiency issues, the number of controllers to be applied on the network nodes should be as small as possible. The goal consists in controlling just a fraction of network nodes by adding some local feedback injections to them, which is known as pinning control. This concept was first discussed in Grigoriev et al., ${ }^{17}$ in which pinning control of a spatiotemporal chaos system made out of coupled map lattices was demonstrated.

It must be stressed that the problem here is not only determining the interconnection coupling strength, the control gain, and the control strategy to be applied to the "pinned" nodes but also determining how many, and which nodes need to be controlled in order to achieve the specific synchronization goal. The problem of determining the number and type of nodes to be pinned, also termed as pinning controllability, was discussed in Refs. 18 and 19 where sufficient and necessary conditions for the stability of synchronization of the desired common solution were also given. The problem of selecting the type of nodes that should be pinned in order to improve synchronization performance ${ }^{20}$ by minimizing synchronization time, and driving the system to a desired state is still an open problem; however, it has been proposed by Porfiri et al. ${ }^{21}$ a node-to-node pinning strategy to maximize synchronization performance, i.e., minimize synchronization time. In node-to-node pinning control, just one node is pinned, and at each instant of time multiple of the switching period $T$, a new node is randomly chosen to be pinned. 
In this work, we propose an adaptive node-to-node pinning control strategy that allows us to efficiently drive the network system to a desired synchronized state. In this approach, both the coupling strength among nodes and the pinning control gain are adaptively changed according to well chosen laws that take into account the specificities of the oscillators and the network topology. We are able to prove that our strategy allows the system dynamics converges to the desired dynamical behavior under nondegenerative conditions. Using numerical results, we are able to quantify how efficient our strategy is.

This work proceeds as follows: In Sec. II, we present our adaptive pinning strategy and show the arguments that allow us to state under which conditions the system dynamics converges to the desired dynamics. In Sec. III, we present results obtained from numerical experiments that numerically support our results. Finally, we end this work with general considerations.

\section{ADAPTIVE PINNING CONTROL STRATEGY}

Let us now, consider the controlled network

$$
\begin{aligned}
\dot{\mathbf{x}}_{i}(t)= & f\left(\mathbf{x}_{i}(t), t\right)-\sum_{j \in \mathcal{N}_{i}} \sigma_{i j} \Gamma\left\{\mathbf{x}_{i}(t)-\mathbf{x}_{j}(t)\right\} \\
& -\delta_{i} q_{i}\left\{\mathbf{x}_{i}(t)-\mathbf{x}_{\mathbf{s}}(t)\right\}
\end{aligned}
$$

$i=1, \ldots, N$, in which the last term is the pinning control term, $q_{i}$ is the control gain of node $i ; \sigma_{i j}$ is the coupling strength between nodes $i$ and $j ; N_{\text {pin }}$ is the number of pinned nodes; $\mathbf{x}_{\mathrm{S}}$ is the desired time continuous synchronous solution to be achieved $-\mathbf{x}_{\mathrm{s}}$ is also a solution of $f(\mathbf{x}(t), t)$ (that we call reference node) being a equilibrium point, a periodic or chaotic orbit.

Let us consider, without loss of generality, that $M_{\text {pin }}$ nodes of the network are chosen as the "pinnable" nodes so that just $N_{\text {pin }}$ nodes randomly chosen are effectively pinned at a given time instant $k T$ (notice that $\left.M_{\text {pin }}>N_{\text {pin }}\right)$.

We define the function $\delta_{i}(t)=\delta_{i}(k T)$ as follows:

$$
\delta_{i}(t)=\left\{\begin{array}{lr}
1, & \text { if } i \in M_{\text {pin }} \text { and } \sum_{i} \delta_{i} \leq N_{\text {pin }}, \\
0, & \text { otherwise. }
\end{array}\right.
$$

in which $M_{\text {pin }}$ is the set of all pinnable nodes.

Suppose the coupling strength and control gain can be adaptively set via adaptation laws given by Eqs. (4) and (5), respectively.

$$
\dot{\sigma}_{i j}(t)=\alpha\left\|\mathrm{e}_{i j}^{\mathrm{c}}(t)\right\|^{p}, \quad \forall(i, j) \in \mathcal{E}, j>i, \quad 0<p \leq 2
$$

in which $\mathbf{e}_{i j}^{\mathrm{c}}(t)=\mathbf{x}_{i}(t)-\mathbf{x}_{j}(t)$ represents nodes synchronization error, $\alpha>0$, and $\mathcal{E}=\cup \mathcal{N}_{i}$. Observe $\dot{\sigma}_{i j}(t)$ is only defined for $j>i$, since, by the symmetry, $\sigma_{j i}(t)=\sigma_{i j}(t)$.

$$
\dot{q}_{i}(t)=\kappa\left\|\mathbf{e}_{i}^{\mathrm{p}}(t)\right\|^{p}, \quad 0<p \leq 2,
$$

in which $\mathbf{e}_{i}^{\mathrm{p}}(t)=\mathbf{x}_{i}(t)-\mathbf{x}_{\mathrm{s}}(t)$ represents pinning control error, $\kappa>0$, where $i=1,2, \ldots, M_{\text {pin }}$.
Equations (2)-(5) define the decentralized fully adaptive node-to-node strategy which we propose in this work - the term fully refers to the fact that all parameters $\sigma_{i j}$ and $q$ that determine synchronizability are adaptively estimated and decentralized because the adaptation laws are independent. Next, we prove that this strategy guarantees global asymptotic stability of the synchronized solution $\mathbf{x}_{\mathrm{s}}(t)$; and we show, via numerical analysis, that such strategy can present better performance than the original decentralized fully adaptive strategy. Different from the decentralized fully adaptive node-to-node strategy, in the decentralized fully adaptive strategy, a network node, or a set of network nodes $M_{\text {pin }}$, is randomly chosen but does not change in time, i.e., $\delta_{i}(t)=\delta_{i}$ defined at the initial time.

Theorem 1. Assuming $\mathrm{f}$ is $\operatorname{QUAD}(\Delta, \bar{\omega})$ (see Definition 3 in the Appendix), with $\Delta-\bar{\omega} I<0$, that the decentralized fully adaptive node-to-node pinning control strategy given by Eqs. (2)-(5)) guarantees global asymptotic synchronization of a connected undirected network onto the desired trajectory $\mathbf{x}_{\mathrm{s}}$ according to Definition 4 . Moreover, both the coupling and control gains converge towards finite values.

\section{A. Proof of Theorem 1}

Based on Ref. 22, the proof of the theorem is based on choosing an appropriate Lyapunov function, and then showing that

1. The derivative $\dot{V}$ of the candidate Lyapunov function $V$ is negative semidefinite (NSD).

2. The various coupling strengths $\sigma_{i j}(t)$ and the pinning control strengths $q_{i}(t)$ are bounded.

3. The pinning errors $\mathbf{e}_{i}^{p}(t) \rightarrow 0$.

Let us define the $N$-dimensional row matrix $\Lambda(t)=$ $\left[\Lambda_{i j}(t)\right]$ as

$$
\Lambda_{i j}(t)=\left\{\begin{array}{cc}
\sigma_{i j}(t), & \text { if }(i, j) \in \mathcal{E}, \\
-\sum_{k \in \mathcal{N}_{i}} \sigma_{i k}(t), & \text { if } i=j, \\
0, & \text { otherwise }
\end{array}\right.
$$

with $\sigma_{i j}(t)=\sigma_{j i}(t)$; and the extended matrix $\Lambda_{\mathrm{E}}(t)$ as the $(N+1)$-dimensional matrix,

$$
\Lambda_{\mathrm{E}}(t)=\left[\begin{array}{ccc} 
& & 0 \\
& \bigwedge & \vdots \\
0 & \cdots & 0
\end{array}\right]
$$

Using definitions given above, the governing equations of the pinned network (2) can be recast as

$$
\dot{X}(t)=F(X(t), t)+\Lambda_{\mathbf{E}}(t) X(t)-\Lambda_{\text {pin }}(\mathbf{t}) X(t), \quad X(0)=X_{0},
$$

where $\quad X(t)=\left[\mathbf{x}_{1}^{T}(t), \ldots, \mathbf{x}_{N}^{T}(t), \mathbf{x}_{\mathrm{s}}^{T}(t)\right]^{T}, \quad F(X(t), t)=\left[f\left(\mathbf{x}_{1}\right.\right.$ $\left.(t), t)^{T}, \ldots, f\left(\mathbf{x}_{N}(t), t\right)^{T}, f\left(\mathbf{x}_{\mathrm{s}}(t), t\right)^{T}\right]^{T}, \quad \Lambda_{\mathbf{E}}(t)=\Lambda_{\mathrm{E}}(t) \quad \otimes I_{n}$, $\Lambda_{\text {pin }}(t)=\Lambda_{\text {pin }}(t) \otimes I_{n}$, in which, 


$\left[\begin{array}{cccccccc}q_{1}(t) \delta_{1}(t) & 0 & \cdots & \cdots & \cdots & \cdots & 0 & -q_{1}(t) \delta_{1}(t) \\ 0 & q_{2}(t) \delta_{2}(t) & 0 & \cdots & \cdots & \cdots & 0 & -q 2(t) \delta_{2}(t) \\ 0 & 0 & \ddots & 0 & \cdots & \cdots & 0 & \vdots \\ 0 & 0 & 0 & q_{M_{\text {pin }}}(t) \delta_{M_{\text {pin }}}(t) & 0 & \cdots & 0 & -q_{M_{\text {pin }}}(t) \delta_{M_{\text {pin }}}(t) \\ 0 & 0 & 0 & 0 & 0 & \cdots & 0 & 0 \\ \vdots & \vdots & \vdots & \vdots & \vdots & \vdots & \vdots & \vdots \\ 0 & 0 & 0 & 0 & 0 & 0 & 0 & 0\end{array}\right]$

is the $(N+1)$-dimensional Laplacian-like matrix $\Lambda_{\text {pin }}(t)$. Without loss of generality, since a Laplacian-like matrix can always be rearranged so that the first $M_{\text {pin }}$ nodes are chosen to be the pinnable nodes.

The edge-based coupling strength adaptation law embedded in $\Lambda_{\mathrm{E}}(t)$ is given by Eq. (4) and the control gain adaptation law is given by Eq. (5), with $\sigma_{i j}(0) \geq 0, \forall(i, j) \in$ $\mathcal{E}$ and $q_{i}(0) \geq 0, \forall i=1, \ldots, M_{\text {pin }}$.

The synchronization error can now be written as $\mathbf{e}(t)=X^{T}(t) \mathbf{M X}(t)$, and synchronization will be achieved if $\lim _{t \rightarrow \infty} \mathbf{e}(t)=0$, i.e., if $\lim _{t \rightarrow \infty} \mathbf{e}_{i j}^{\mathrm{c}}(t)=\mathbf{0}$ and $\lim _{t \rightarrow \infty} \mathbf{e}_{i}^{\mathrm{p}}(t)$ $=\mathbf{0}$. To prove this to be the case, we can consider the following candidate Lyapunov function:

$$
\begin{aligned}
V(X, \sigma, \mathbf{q}, t)= & \frac{1}{2} \eta X^{T}(t) \mathbf{M} X(t)+\frac{1}{2 \gamma}[\mathbf{c}-\sigma(t)]^{T}[\mathbf{c}-\sigma(t)] \\
& +\frac{1}{2 \beta}[\mathbf{r}-\mathbf{q}(t)]^{T}[\mathbf{r}-\mathbf{q}(t)],
\end{aligned}
$$

where $\eta, \gamma$, and $\beta$ are positive scalars, $\mathbf{r}$ is an $M_{\text {pin }}$-dimensional arbitrary vector, $\mathbf{c}$ is an $m$-dimensional arbitrary vector, $\sigma(t)=\left\{\sigma_{i}\right\}(t)$ is defined as the vector whose elements are all the adaptive coupling gains among nodes, that is, $\sigma(t)$ is the vector $\left[\sigma_{11}(t) \sigma_{12}(t) \cdots\right]$ of size $m=|\mathcal{E}|, \mathbf{q}(t)=$ $\left\{q_{i}(t)\right\}$ is defined as the vector whose elements are the pinning control gains.

The $(N+1)$-dimensional matrix $M$ is defined as follows:

$$
M=\left[\begin{array}{rrrc} 
& & & -1 \\
& I_{N} & & \vdots \\
& & & -1 \\
-1 & \cdots & -1 & N
\end{array}\right],
$$

also define $\mathbf{M}=M \otimes I_{n}$.

We then have

$$
\begin{aligned}
\dot{V}= & \eta X^{T}(t) \mathbf{M}\left[F(X(t), t)+\Lambda_{\mathbf{E}}(t) X(t)-\Lambda_{\mathbf{p i n}}(t) X(t)\right] \\
& -\frac{1}{\gamma} \sum_{i=1}^{m}\left(c_{i}-\sigma_{i}(t)\right)^{T} \dot{\sigma}_{i}(t)-\frac{1}{\beta} \sum_{i=1}^{M_{\text {pin }}}\left(r_{i}-q_{i}(t)\right)^{T} \dot{q}_{i}(t) \\
= & \eta X^{T}(t) \mathbf{M}[F(X(t), t)-\Delta X(t)]+\eta X^{T}(t) \mathbf{M} \Delta X(t) \\
& +\eta X^{T}(t) \mathbf{M} \Lambda_{\mathbf{E}}(t) X(t)-\eta X^{T}(t) \mathbf{M} \Lambda_{\text {pin }}(t) X(t) \\
& -\frac{1}{\gamma} \sum_{i=1}^{m}\left(c_{i}-\sigma_{i}(t)\right)^{T} \dot{\sigma}_{i}(t)-\frac{1}{\beta} \sum_{i=1}^{M_{\text {pin }}}\left(r_{i}-q_{i}(t)\right)^{T} \dot{q}_{i}(t),
\end{aligned}
$$

with $\Delta=\Delta \otimes I_{n}$. Since $f$ is QUAD by assumption, we can state that $X^{T}(t) \mathbf{M}[F(X(t), t)-\Delta X(t)] \leq-\bar{\omega} X^{T}(t) \mathbf{M} X(t)$, so that

$$
\begin{aligned}
\dot{V} \leq & -\eta \bar{\omega} X^{T}(t) \mathbf{M} X(t)-\eta X^{T}(t) \mathbf{M} \Lambda_{\mathbf{p i n}}(t) X(t) \\
& +\eta X^{T}(t)\left[\mathbf{M} \Delta+\mathbf{M} \Lambda_{\mathbf{E}}(t)\right] X(t) \\
& -\frac{1}{\gamma} \sum_{i=1}^{m}\left(c_{i}-\sigma_{i}(t)\right)^{T} \dot{\sigma}_{i}(t) \frac{1}{\beta} \sum_{i=1}^{M_{\mathrm{pin}}}\left(r_{i}-q_{i}(t)\right)^{T} \dot{q}_{i}(t) .
\end{aligned}
$$

Equation (9) can also be written as

$$
\begin{aligned}
\dot{V} \leq & -\eta X^{T}(t) \mathbf{M} \Lambda_{\mathbf{p i n}}(t) X(t)+\eta X^{T}(t)[\mathbf{M}(\Delta-\bar{\omega} \mathbf{I}) \\
& \left.+\mathbf{M} \Lambda_{\mathbf{E}}(t)\right] X(t)-\frac{1}{\gamma} \sum_{\mathcal{E}}\left[c_{i j}-\sigma_{i j}(t)\right] \varphi\left(\mathbf{x}_{i}(t)-\mathbf{x}_{j}(t)\right) \\
& -\frac{1}{\beta} \sum_{\mathcal{P}}\left(r_{i}-q_{i}(t)\right) \vartheta\left(\mathbf{x}_{i}(t)-\mathbf{x}_{s}(t)\right) \\
:= & W_{1}(X(t), \mathbf{q}(t))+W_{2}(X(t), \sigma(t))+W_{3}(X(t), \sigma(t)) \\
& +W_{4}(X(t), \mathbf{q}(t)) .
\end{aligned}
$$

One can easily check that the matrix $M \Lambda_{\mathrm{E}}(t)=\Lambda_{\mathrm{E}}(t)$, and matrix $M \Lambda_{\text {pin }}(t)$ will be given by

$$
\left[\begin{array}{cccccccc}
q_{1}(t) \delta_{1}(t) & 0 & \cdots & \cdots & \cdots & \cdots & 0 & -q_{1}(t) \delta_{1}(t) \\
0 & q_{2}(t) \delta_{2}(t) & 0 & \cdots & \cdots & \cdots & 0 & -q 2(t) \delta_{2}(t) \\
0 & 0 & \ddots & 0 & \cdots & \cdots & 0 & \vdots \\
0 & 0 & 0 & q_{M_{\mathrm{pin}}}(t) \delta_{M_{\mathrm{pin}}}(t) & 0 & \cdots & 0 & -q_{M_{\mathrm{pin}}}(t) \delta_{M_{\mathrm{pin}}}(t) \\
0 & 0 & 0 & 0 & \cdots & 0 & 0 \\
\vdots & \vdots & \vdots & \vdots & \vdots & \vdots & \vdots & \vdots \\
-q_{1}(t) \delta_{1}(t) & -q_{2}(t) \delta_{2}(t) & \cdots & -q_{M_{\mathrm{pin}}}(t) \delta_{M_{\mathrm{pin}}}(t) & 0 & 0 & 0 & \sum_{i=1}^{M_{\mathrm{pin}}} q_{i}(t) \delta_{i}(t)
\end{array}\right] .
$$


The matrices $M \Lambda_{\text {pin }}(t)$ and $M \Lambda_{\mathrm{E}}(t)$ are therefore both symmetric and real for any time $t \geq 0$. From Definition 1, $M \Lambda_{\text {pin }}(t)$ and $-M \Lambda_{\mathrm{E}}(t)$ are also diagonally dominant (see Ref. 23) and, having non-negative diagonal entries. Then, Lemma 1 yields $M \Lambda_{\text {pin }}(t)$ is positive semidefinite (PSD) and so is $-M \Lambda_{\mathrm{E}}(t)$ (i.e., $M \Lambda_{\mathrm{E}}(t)$ is NSD). Thus, in Eq. (10), $W_{1}(X(t), \mathbf{q}(t)) \leq 0$ for any time $t \geq 0$. From the assumption that $\Delta-\bar{\omega} I<0$ is real symmetric, we have $M(\Delta-\bar{\omega} I) \leq 0$. Therefore, $\left[\mathbf{M}\left(\Delta-\bar{\omega} \mathbf{I}+\Lambda_{\mathbf{E}}(t)\right)\right] \leq 0$ and so we have that $W_{2}(X(t), \sigma(t)) \leq 0$ in Eq. (10) for any time $t \geq 0$. We now have to look at the terms $W_{3}(X(t), \sigma(t))$ and $W_{4}(X(t), \mathbf{q}(t))$ in Eq. (10).

We can write $W_{3}(X(t), \sigma(\mathbf{t}))=\sum_{\mathcal{E}}\left(c^{i}-\sigma^{i}(t)\right)\left\|\mathbf{e}_{i j}^{\mathrm{c}}(t)\right\|^{p}$ and $W_{4}(X(t), \mathbf{q}(t))=\sum_{\mathcal{P}}\left(r_{i}-q_{i}(t)\right)\left\|\mathbf{e}_{i}^{\mathrm{p}}(t)\right\|^{p}$. Functions $W_{2}(X(t), \sigma(t))$ and $W_{3}(X(t), \sigma(t))$ in Eq. (10) are both linear functions of $\sigma_{i}(t)$; similarly, $W_{1}(X(t), \mathbf{q}(t))$ and $W_{4}(X(t)$, $\mathbf{q}(t))$ are both linear functions of $q_{i}(t)$. Hence, if $\sigma_{i}(t)$ and $q_{i}(t)$ diverged in time, these terms would diverge exponentially. Nevertheless, there will always exist a suitable value of the constants $\eta, \gamma$, and $\beta$ such that, for all $X(t), \sigma_{i}(t)$ and $q_{i}(t),\left|W_{2}(X(t), \sigma(t))\right| \geq\left|W_{3}(X(t), \sigma(t))\right|$, and $\mid W_{1}(X(t), \mathbf{q}(t))$ $|\geq| W_{4}(X(t), \mathbf{q}(t)) \mid$ for any time $t \geq 0$. As $W_{1}(X(t), \mathbf{q}(t))$ is NSD and $W_{2}(X(t), \sigma(t))$ is NSD, we get for all $X(t), \sigma_{i}(t)$ and $q_{i}(t), \dot{V} \leq 0$ against the assumption that $\sigma_{i}(t)$ and $q_{i}(t)$ diverge in time. Therefore, the various $\sigma_{i}(t)$ and $q_{i}(t)$ are upper bounded and $\dot{V} \leq 0$ for all $X(t) \in \mathbb{R}^{n(N+1)}$, $\sigma_{i}(t) \in \mathbb{R} \forall i=1, \ldots, m, q_{i}(t) \in \mathbb{R} \forall i=1, \ldots, M_{\text {pin }}$; and the synchronization error $\mathbf{e}(t)=X(t)^{T} \mathbf{M} X(t)$ is bounded.

Therefore, we can conclude that $\dot{V} \leq 0$ and so both the error $\mathbf{e}(t)$ and the coupling and control gains $\sigma_{i j}(t)$ and $q_{i}(t)$ are bounded.

From Eqs. (4) and (5), we know that all the gains are strictly monotonically increasing and so, being bounded, they converge to constant values. Therefore, asymptotically $\dot{\sigma}_{i j}(t) \rightarrow 0, \forall(i, j) \in \mathcal{E}$ and $\dot{q}_{i}(t) \rightarrow 0, \forall i=1, \ldots, N$. From Eq. (9), we can finally conclude that the error $\mathbf{e}(t)$ must asymptotically converge to zero.

\section{NUMERICAL RESULTS}

In this section, we present an example of the decentralized fully adaptive node-to-node pinning control applied to a complex network of 300 Chen's oscillators. ${ }^{24}$ We also compare the performance of the new strategy to the decentralized fully adaptive strategy.

Consider a network of 300 nodes structured on a scalefree like topology with 2400 links constructed using the BA model presented in Ref. 25. Specifically, we consider a controlled network of the form (2) consisting of 300 identical Chen's oscillators defined as follows:

$$
\dot{\mathbf{x}}_{i}=\left(\begin{array}{ccc}
-r_{1} & r_{1} & 0 \\
0 & r_{3} & 0 \\
0 & 0 & r_{2}
\end{array}\right)\left(\begin{array}{c}
x_{i 1} \\
x_{i 2} \\
x_{i 3}
\end{array}\right)+\left(\begin{array}{c}
0 \\
-x_{i 1} x_{i 3} \\
x_{i 1} x_{i 2}
\end{array}\right),
$$

where $i=1, \ldots, 300, r_{1}=35, r_{2}=3, r_{3}=28$, parameters for which the system exhibits chaotic behavior.

Initial conditions on network nodes are randomly selected from a normal distribution with mean $(\sqrt{63}, \sqrt{63}, 21)$ and
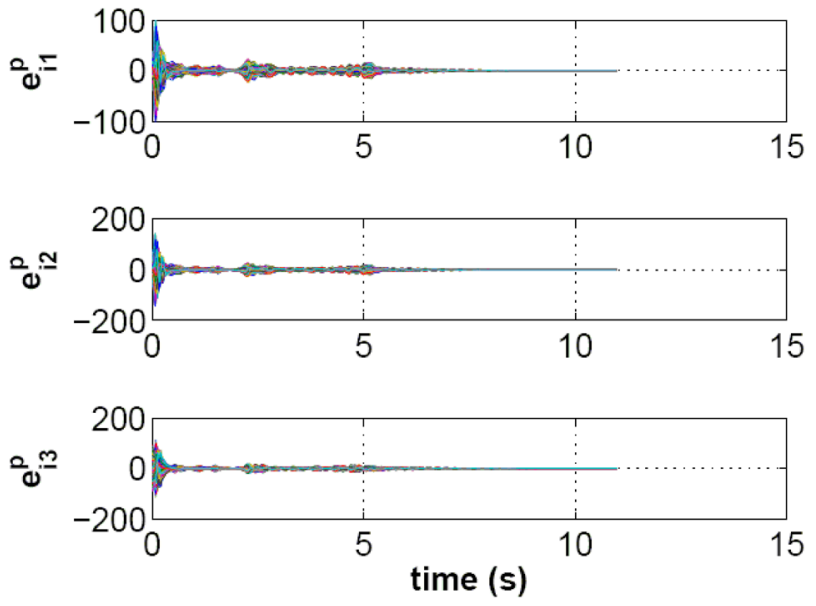

FIG. 1. Pinning error $\mathbf{e}_{i}^{p}(t)$ : Figure shows the evolution of the pinning synchronization error $\mathbf{e}_{i}^{p}(t)$ of each state variable of each node of the network. Observe states' error go to zero as time evolves. In the figure, each different color represents one node different node of the network.

standard deviation 1. Initial condition of the reference node is $(\sqrt{63}, \sqrt{63}, 21)$, that is an unstable equilibrium point. The total number of pinned nodes, $N_{\text {pin }}$, is set to 1 ; pinned nodes are chosen by random each instant of time multiple of the switching period $T=0.05$ unit time. Initial conditions of parameters $\sigma_{i j}$ and $q$ are all zero.

Figure 1 shows error time evolution of the network nodes when applying decentralized fully adaptive node-to-node pinning control strategy, observe that error $\mathbf{e}_{i}^{\mathrm{p}}(t)$ evolves toward zero.

Figure 2 shows the time evolution of the states of the network, observe node states synchronize onto the reference orbit that is an equilibrium point in this case. Figure 3 shows the evolution of the coupling strength $\sigma_{i j}(t)$, observe they all converge to low constant values. Finally, Figure 4 shows time evolution of the control gain $q_{j}(t)$ of the pinned node $j$, observe the switching behaviour of the curve due to the characteristic of the control strategy.
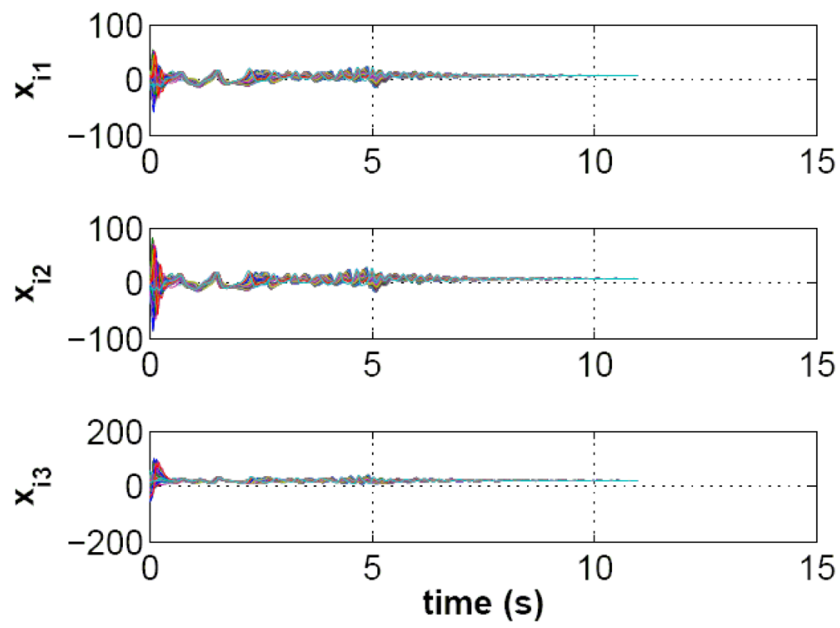

FIG. 2. Synchronous state: Figure shows the time evolution of each state variable of each node of the network. Observe states go to the equilibrium point set by the reference node as time evolves. In the figure, each different color represents one node different node of the network. 


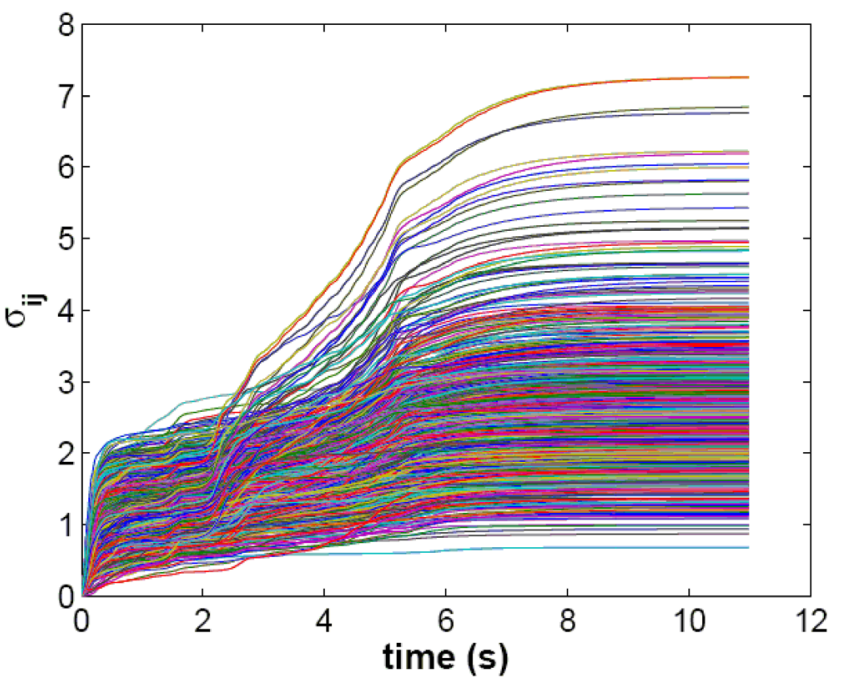

FIG. 3. Coupling strength $\sigma_{i j}(t)$ : Figure shows the evolution of each coupling strength $\sigma_{i j}(t)$ of the network edges (links). Observe each coupling strength converges to a bounded value as time evolves. In the figure, each different color represents one node different coupling strength $\sigma_{i j}(t)$.

We now compare the performance of the decentralized fully adaptive node-to-node pinning control to the original decentralized fully adaptive strategy. To do that, we compare the convergence time of the two strategies averaged over 50 simulation realizations. The convergence time is defined as the time the Euclidian norm of the pinning error $\mathbf{e}^{\mathrm{p}}(t)$ takes to be less than $\epsilon=\sqrt{0.001}$. The result is an average convergence time of 16.29 units of time for the decentralized fully adaptive strategy in which the node to be pinned is chosen by random at initial time, while the decentralized fully adaptive node-to-node pinning strategy has an average convergence time of 14.64 units of time, confirming the proposed strategy can have better performance than the original decentralized fully adaptive strategy. The numerical experiment can be also performed on a random or small-world network

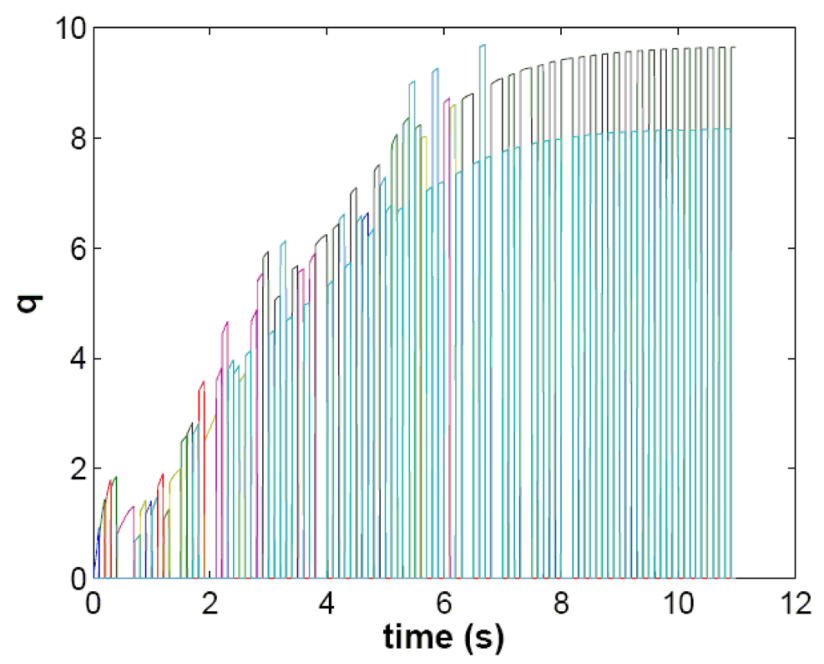

FIG. 4. Pinning control gain $q(t)$ : Figure shows the evolution of the pinning control gain $q_{j}(t)$ of the pinned node $j$. Observe each control gain $q_{j}(t)$ converges to a bounded value as time evolves. In the figure, each different color represents the control gain of a different pinnable node. preserving the qualitative result. We do not show it in this paper for a matter of brevity.

\section{CONCLUSION}

When one deals with a pinning control strategy for the synchronization of complex network two characteristics are highly desirable: online estimation of the coupling strength and the control gain necessary to guarantee synchronization; and minimization of the convergence time. In this work, we propose a strategy that has both desirable characteristicsthe decentralized fully adaptive node-to-node strategy for the synchronization of complex networks.

In this work, we define the decentralized fully adaptive node-to-node strategy; we prove the proposed strategy guarantees global asymptotic stability of the synchronized solution $\mathbf{x}_{s}$; and we show, via numerical analysis, that such strategy can present better performance than an adaptive strategy like the decentralized fully adaptive strategy.

We would like to thank FAPESP and CNPq for the financial support to this work.

\section{APPENDIX: MATHEMATICAL DEFINITIONS}

We report here some mathematical definitions and lemmas that are used in the paper.

Definition 1. Let A be an m-dimensional square matrix. According to Ref. 26, the matrix A is said to be diagonally dominant if

$$
\left|a_{i i}\right| \geq \sum_{j=1, j \neq i}^{m}\left|a_{i j}\right|
$$

Lemma 1. Let A be a Hermitian m-dimensional diagonally dominant square matrix with non-negative diagonal entries. Then, A is PSD. By Gershgorin's circle theorem, ${ }^{26}$ for each eigenvalue $\lambda$ of $\mathrm{A}$, an index $i$ exists such that

$$
\lambda \in\left[a_{i i}-\sum_{j=1, j \neq i}^{m} a_{i j}, a_{i i}+\sum_{j=1, j \neq i}^{m} a_{i j}\right],
$$

which implies, from Definition 1 , that $\lambda_{j} \geq 0, \forall j=1, \ldots, m$.

Definition 2. The Laplacian m-dimensional square matrix A of a connected undirected network is a symmetric irreducible PSD matrix, with $\operatorname{rank}(A)=m-1$, defined by

$$
A=\left\{\begin{array}{ccc}
-1, & \text { if } & (i, j) \in \mathcal{E}, \\
-\sum_{k \in \mathcal{N}_{i}} a_{i k}, & \text { if } & i=j, \\
0, & \text { otherwise. }
\end{array}\right.
$$

where $\mathcal{E}$ is the set off all connected nodes.

Definition 3. A function $f: \mathbb{R}^{n} \times \mathbb{R}^{+} \rightarrow \mathbb{R}^{n}$ is $\operatorname{QUAD}(\Delta, \bar{\omega})$, see Ref. 27, if, for any $x, y \in \mathbb{R}^{n}$,

$$
(\mathbf{x}-\mathbf{y})^{T}[f(\mathbf{x}, t)-f(\mathbf{y}, t)] \leq(\mathbf{x}-\mathbf{y})^{T}(\Delta-\bar{\omega} I)(\mathbf{x}-\mathbf{y})
$$

where $\Delta$ is an arbitrary diagonal matrix of order $n$, and $\bar{\omega}$ is a positive scalar. 
Definition $4 .^{24}$ Let $\mathbf{x}_{i}\left(t ; t_{0}, X_{0}\right), i=1, \ldots, N$, be a solution of the controlled network (2), where $X_{0}=\left(\mathbf{x}_{1}^{T}(0), \ldots\right.$, $\left.\mathbf{x}_{N}^{T}(0)\right)^{T} \in \mathbb{R}^{n N}$. Let $f: \Omega \times \mathbb{R}^{+} \rightarrow \mathbb{R}^{n}$ be continuously differentiable, $\Omega \subseteq \mathbb{R}^{n}$. If there exists a nonempty subset $\Gamma \subseteq \Omega$, with $\mathbf{x}(0) \in \Gamma, i=1, \ldots, N$, such that $\mathbf{x}_{i}\left(t ; t_{0}, X_{0}\right)$ $\in \Omega, i=1, \ldots, N$, for all $t \geq t_{0}$, and

$$
\lim _{t \rightarrow \infty}\left\|\mathbf{x}_{i}\left(t ; t_{0}, X_{0}\right)-\mathbf{x}_{\mathrm{s}}\left(t ; t_{0}, \mathbf{x}_{\mathrm{s}}(0)\right)\right\|_{2}=0, \quad i=1, \ldots, N
$$

where $\mathbf{x}_{\mathrm{s}}(0) \in \Omega$, then the controlled network (2) is said to achieve controlled network synchronization and $\Gamma \times \ldots \times \Gamma$ is called the region of synchrony for the dynamic network (2).

To achieve controlled network synchronization, the control $-q_{i}(t) \mathbf{e}_{i}^{\mathrm{p}}(t)$ should make the norm of all error vectors $\mathbf{e}_{i}^{\mathrm{p}}(t)$ approach zero as time $\mathrm{t}$ goes to infinity. That is

$$
\lim _{t \rightarrow \infty}\left\|\mathbf{e}_{i}^{\mathrm{p}}(t)\right\|_{2}=0, \quad i=1, \ldots, N
$$

${ }^{1}$ J. Cortes, S. Martinez, and F. Bullo, IEEE Trans. Autom. Control 51, 1289 (2006).

${ }^{2}$ D. A. Paley, N. E. Leonard, R. Sepulchre, D. Grunbaum, and J. K. Parrish, IEEE Control Syst. Mag. 27, 89 (2007).

${ }^{3}$ D. A. Paley, N. E. Leonard, and R. Sepulchre, Syst. Control 57, 209 (2008).

${ }^{4}$ F. Sorrentino and E. Ott, Phys. Rev. Lett 100, 114101 (2008).

${ }^{5}$ A. B. Cohen, B. Ravoori, F. Sorrentino, T. E. Murphy, E. Ott, and R. Roy, Chaos 20, 043142 (2010).
${ }^{6} \mathrm{~N}$. E. Leonard and E. Fiorelli, in Proceedings of the 40th IEEE Conference on Decision and Control 2001 (IEEE, 2001), Vol. 3, pp. 2968-2973.

${ }^{7}$ R. Olfati-Saber and R. M. Murray, IEEE Trans. Autom. Control 49(9), 1520 (2004).

${ }^{8}$ I. D. Couzin, J. Krause, N. R. Franks, and S. A. Levin, Nature 433, 513 (2005).

${ }^{9}$ B. Nabet, N. E. Leonard, I. D. Couzin, and S. A. Levin, J. Nonlinear Sci. 19, 399 (2009).

${ }^{10}$ G. Orosz, J. Moehlis, and R. M. Murray, Philos. Trans. R. Soc. A 368, 439 (2010).

${ }^{11}$ W. Wang and J. -J. E. Slotine, IEEE Trans. Autom. Control 51(7), 1156 (2006).

${ }^{12}$ L. M. Pecora and T. L. Caroll, Phys. Rev. Lett. 64, 821 (1990).

${ }^{13}$ C. W. Wu and L. O. Chua, IEEE Trans. Circuits Syst. I: Fundam. Theory Appl. 42, 430 (1995).

${ }^{14}$ T. Nishikawa and A. E. Motter, Physica D 224, 77 (2006).

${ }^{15}$ J. Sun, E. M. Bollt, and T. Nishikawa, Complex Sciences (Springer, Berlin, 2009), Vol. 4, pp. 900-911.

${ }^{16}$ L. M. Pecora and T. L. Caroll, Phys. Rev. Lett. 80, 2109 (1998).

${ }^{17}$ R. Grigoriev, M. C. Cross, and H. G. Schuster, Phys. Rev. Lett. 79, 2795 (1997).

${ }^{18}$ F. Sorrentino, M. di Bernardo, F. Garofalo, and G. Chen, Phys. Rev. E 75, 046103 (2007).

${ }^{19}$ M. Porfiri and M. di Bernardo, Automatica 44, 3100 (2008).

${ }^{20}$ L. F. R. Turci and E. E. N. Macau, Phys. Rev. E 84, 011120 (2011).

${ }^{21}$ M. Porfiri and F. Fiorilli, Chaos 19, 013122(11) (2009).

${ }^{22}$ K. J. Astrom and B. Wittenmark, Adaptive Control (Addison-Wesley Reading, 1994).

${ }^{23}$ P. De Lellis, M. di Bernardo, and F. Garofalo, Chaos 18, 037110 (2008).

${ }^{24}$ J. Zhou, J. Lu, and J. Lü, Automatica 44, 996 (2008).

${ }^{25}$ A.-L. Barabási and R. Albert, Science 286, 509 (1999).

${ }^{26}$ R. A. Horn and C. R. Johnson, Matrix Analysis (Cambridge University Press, 1985).

${ }^{27}$ W. Lu and T. Chen, Physica D 213, 214 (2006). 